\title{
Anxiety and the COVID-19 Pandemic
}

\author{
Avinash De Sousa \\ Consultant Psychiatrist and Founder Trustee, Desousa Foundation, Mumbai. \\ Corresponding author - Avinash De Sousa \\ E-mail - avinashdes888@gmail.com
}

\begin{abstract}
The COVID-19 pandemic with its widespread global impact on health, healthcare infrastructure and the psycho-social and economic disruption has never been seen in modern times. The virus causing it is a new one and hence not only do we not know the optimum management of it [1]. After a year of the pandemic, we are yet struggling to overcome it. It is rare in a pandemic where a vaccine has been synthesized and manufactured within a year and now with different more infectious variants spreading and the second, third, fourth and fifth waves in some countries (USA, Canada) we seem to be in the learning curve with this pandemic. Considering the proportion of the pandemic affecting almost all the countries in the world and some of them having high morbidity rates, the fear \& anxiety is high [2]. There is a tremendous amount of information/misinformation that is so easily available in the social media. The psychological complications are increasing rapidly. A year into the pandemic and we are facing the second wave in India which seems to be worse than the first. Unlike the first wave we now seem to be facing fatigue not only in the general population but more so in the healthcare and frontline workers. There are various forms of anxiety that have been encountered during the pandemic and we look at various forms of anxiety from an Indian perspective.
\end{abstract}

\section{COVID Related Anxiety or Corona-Anxiety}

COVID related anxiety is a major form of anxiety. Especially during the early days of the pandemic, when India was under lockdown, individuals faced major anxiety at the thought of themselves or their families being infected. Popularly known as 'coronaphobia' this fear of the virus itself causes a lot of anxiety over the possibility of being hospitalized, or even dying. Media information via news channels and social media have witnessed a lot of sensationalism and even fake news. This constant access to news updates about rising numbers, buildings being sealed and first-hand accounts of hospitals are a source of anxiety and stress. The advent of social media journalism has led to fake news that does not come from credible sources [3-4].

\section{Health Anxiety related to procuring COVID-19}

Health anxiety denotes a continuum of behaviors and cognitions of an individual that can range from persistent worries about illness which may vary from mild to severe forms bordering on hypochondriasis. Compulsory contact tracing and long periods of quarantine which are measures taken to control the epidemic increase people's anxiety and the remorse regarding passing on the infection and its consequences along with the stigma attached. Health anxiety has led to everyone attributing routine cold and cough to COVID and getting themselves tested when there is no need [5].

\section{Work from home induced Anxiety}

Work from home is not a new concept but has been enforced on a large population with the exception of healthcare professionals and frontline workers. The enforced stay at home and work from home along with the presence of other family members which may include other working members, the elderly and school going children or infants and pre-schoolers. This may lead to overcrowding, interpersonal strained relationships, a severe crunch of resources especially if multiple members are working from home for laptops and smartphones used for office and school work. The reduction in pay, difficulty in working from home while looking after other family members, elderly and children may add to the stressors especially 
for women. The skills needed to work from home may not be good and entails new learning and training which can prove to be anxiety provoking [6].

\section{Quarantine Anxiety}

One of the preventive measures for COVID-19 infection is quarantine for a period ranging from 14 days and more depending on the presence of symptoms and Covid test results. This becomes an extremely stressful period with different coping mechanisms employed individually. Being isolated and alone during an extremely vulnerable period of illness increases the chances of anxiety and depressive disorders, substance use disorders and PTSD. The loneliness and uncertainty regarding prognosis, financial burden and worries about the health of the near and dear ones leaves one emotionally and physically vulnerable [7].

\section{COVID Recovery Anxiety}

Patients who were in the ICU reported post COVID complications like reduced lung functioning and reduced exercise capacity. In the ICU, panic disorder and specific anxiety disorders like claustrophobia are seen. The loss of human touch in the ICU with healthcare professionals being in PPE kits leads to extreme loneliness and feelings of helplessness in an excruciatingly vulnerable state. The survivors may have psychological and neurological sequelae due to the prolonged ICU stay. Stressors include immobility, separation from family and friends, pronounced sedation, anxiety about health and survival and financial concerns. The prolonged time taken to recover from post-covid complications adds to the anxiety along with the fear of reinfection [8].

\section{Generalized Anxiety Disorder, Panic and Pre-Existing Anxiety}

From quarantine, to the impact of media and statistics, there are a number of factors that can impact those who have been diagnosed with generalized anxiety disorder, panic attacks and phobias such as germophobia. For example, an individual with general anxiety who tests COVID-10 positive and has to be quarantined in a room away from others can have very severe complications in addition to the physical diagnosis of COVID [9]. With jobs lost during the pandemic, financial insecurity can also be a massive anxiety provoking factor, especially among those who rely on daily wages for mere survival. The diagnosis of COVID, along with job insecurities has led to an increase in the reporting of panic attacks in India [10]. COVID-19 related fear can also induce panic attacks. The thoughts of a loved one getting the virus, loss of life, and the fear of contracting the virus are all anxious thoughts that can have disastrous impacts.

\section{Anxiety in patients with pre-existing psychiatric disorders}

Patients with mental health disorders are more vulnerable to stress related events and find it difficult to cope with their original disorder along with the negative life events. Mental health disorders are a risk factor for developing symptoms like insomnia and anxiety during the pandemic. They also have a higher mortality rate. It is also a fact that mental health disorders are often neglected during a disaster and this makes them more vulnerable compounded by the inability to access healthcare during lock downs. This causes nonadherence to medication causing a relapse or deterioration of the already existing illness [11]. Patients with affective and anxiety disorders are more concerned about external environment than patients with schizophrenia. Hence the increase in anxiety and insomnia in anxious or depressed patients is more likely due to COVID-19 related issues and availability of drugs while in patients with schizophrenia it is more likely due to irregular intake or stopping of medication [12].

\section{Fear and anxiety of losing a loved one}

The fear and anxiety of losing a loved one due to COVID is a facet to take into consideration, because of the sudden trauma faced. Social distancing, quarantine and lockdowns have kept individuals away from their families, and children living away from their aged parents often face the anxiety of them possibly never seeing them again. For the elderly living away from home, there is the fear of dying alone, without any support. The current procedures in India do not allow the family to see the individual in hospital, and the cremation procedures are done by local government bodies. The thought of not cremating the loved 
one who died due to COVID-19 is traumatic, and this trauma can cause a lot of anxious thoughts and reactions. The fear of a family member not having access to life saving resources is an uncontrollable situation and this uncertainty for the family causes a lot of anxiety [13].

\section{COVID-19 and Anxiety in the elderly}

There are a number of elderly individuals who live in cities away from their children. COVID-19 poses a number of risk factors for anxiety for this age group as well. COVID-19 has caused the highest number of deaths among senior citizens due to a number of age-related factors and associated comorbidities. The anxiety of contracting the virus is twofold, because of the fear of dying due to COVID-19, without having anyone to be there for them. Further, hearing about relatives and friends succumbing to the virus can cause a lot of anxiety. Grieving for a spouse, sibling, or a friend who died due to covid once again, can cause long term mental health effects [14]. Another facet of anxiety faced by the elderly population is access to daily necessities and resources. Many of the senior citizens living alone have to visit banks and grocery stores for their day-to-day living. During the lockdown and for safety reasons, the elderly could not venture out of their homes for such basic necessities. This poses a serious risk factor for anxiety, as they are unsure about who will purchase the staple ingredients for them. The advent of technology and the smartphone age has always been a fear that the elderly have faced. However, with COVID, this fear can manifest itself as a form of anxiety.

\section{COVID-19 and Anxiety in Women}

Two factors might be taken into consideration while discussing anxiety among women in India. These two factors are pregnancy, and domestic violence. Within just a few weeks of lockdown being announced in India in April 2020, the National Commission of Women saw a $100 \%$ rise in reported cases of domestic violence [15]. Being at home has led to women being locked inside with their abusers, without any escape. The anxiety caused due to the trauma and pain inflicted can have disastrous impacts on their mental health. There is no way they can have a support system, or register a formal complaint with the police during this time. COVID-19 can also be a particularly anxious time for pregnant women in India. The possibility of being infected at the time of labor, without any support due to hospital measures can be an anxious time, especially in small towns and villages that have limited medical facilities [16].

\section{COVID-19 and Pregnancy Related Anxiety}

Pregnancy is one of the most important events in women's life. It is associated with many physiological, psychological and social changes. They are prone to psychological problems like fatigue, emotional disorders, mood disorders and anxiety disorders. They also have the extra burden and challenges of caring for the other children and family members. Pregnancy related anxiety (PRA) is a common problem during pregnancy. PRA is related to fears and concerns regarding the pregnancy, delivery, neonatal health and childrearing. This can negatively affect women's physical and mental health and their children's overall physical, emotional, behavioral and cognitive development. A major source of PRA is infectious diseases especially during epidemic and pandemics. Epidemics are known to cause negative clinical outcomes in pregnant woman such as death, spontaneous abortion, premature birth and fetal death [17]. During the pandemic, a study showed that $50-71 \%$ women were worried about their health status. They are concerned about their own health $\&$ that of their unborn child, access to healthcare facilities, lack of family \& social support \& quarantine in case they get infected. Due to lockdown there's poor access for prenatal examinations \& many women faced difficulty going to hospitals. The women in 2 nd \& especially the $3 \mathrm{rd}$ trimester showed more signs of anxiety [18].

\section{COVID-19 and anxiety in children, adolescents, and the youth}

When speaking of young children, their childhoods are supposed to be spent outdoors, at school, with friends. However, the advent of the pandemic has made children of all age groups stay at home, with increased hours of screen time. While the government and education ministries have released guidelines to control screen time, online classes bring with them mental health concerns. Among older children and college going students, having access to an electronic device can cause a lot of anxiety. There is a major 
digital divide between various regions of India, and a recent article has revealed that in the state of Maharashtra, only half the students have access to forms of online learning [19]. The inability to attend online classes due to a lack of electricity, or internet connection poses a serious problem to students, as their classes and even exams get hindered. The youth have also developed a more 'relaxed' attitude towards covid 19 as they do not believe that it is dangerous within their age group. As a result, they have violated social distancing norms. The youth can actually have a reverse effect- by feeling anxious due to not meeting friends and peers for long periods of time. This, popularly known by the youth is called FOMO, or the fear of missing out [20].

Children who have had a family member or a friend infected with COVID-19 have higher levels of anxiety and depression. Anxiety symptoms are more in females and in children studying in higher grades. However, for some children especially those suffering from social anxiety disorder remaining at home and online schooling may temporarily relieve their anxiety, however may result in overwhelming anxiety when they have to return back to school [21].

\section{COVID Vaccine Related Anxiety}

Anxiety about COVID 19 vaccine-most vaccines are developed over a few years. However, with COVID19 first being reported just a year ago many people are concerned about if a vaccine developed in such a short time frame can truly be safe. Without years of trials, it is difficult to predict long-term side-effects. The state of the pandemic changes daily and recommendations from government officials fluctuate as well and hence the anxiety regarding vaccines is understandable. The choice $\&$ availability between 2 vaccines coupled with inadequate data leads to a lot of ambiguity and confusion regarding the safety and efficacy of the vaccines. The COVID-19 vaccines have caused a lot of stir, and the initial lack of information has led to speculation, causing anxiety and fear. Information in the news about vaccine related complications has also led people to rethink their choices about which vaccine to take. Recent news about life threatening side effects can cause a lot of anxiety, especially if the individual has taken the particular vaccine in question. In India, the recent shortage of vaccines can be a potential facet of anxiety, given the full blown second wave that the country is facing [21].

\section{Financial and economic anxiety}

Months of lockdown for a developing country's economy has had disastrous impacts on the stock market, businesses, factories and daily wage laborers. The pandemic has put many migrant and blue-collar workers under tremendous pressure. We have already seen that over the last year, most of India's factories had to close temporarily and the only people who were affected were those who were usually doing manual labor. There are many reasons for this increase in stress. The first reason is the volatility of the current economic situation. India has struggled to curb the spread of coronavirus and with a lockdown being the only measure when cases get too high, this means that workers are at the mercy of the lockdown policies. Workers who earn a daily wage are at the most risk of job loss and are also the most vulnerable to new restrictions in movement and trade by the government. This uncertainty of not knowing how they will pay for their next meal can cause massive anxiety that can manifest itself as multiple physical symptoms as well. The second reason why these workers have increased stress is that they are worried about infecting their loved ones at home [22].

\section{Second Wave COVID Anxiety}

India is currently facing a full blown second wave, that is far worse than the first wave. Despite vaccination drives going smoothly, a number of factors have contributed to the second wave. In the state of Maharashtra particularly, there has been the highest spike of cases, accompanied by a shortage of vaccines, oxygen, and hospital beds. With new lockdown like restrictions, the situation arising out of the second wave is one that is likely to cause a lot of anxiety for all groups. Businesses and day to day economic activities are once again going through uncertainty, students are facing anxiety over announcement of exams and admission procedures, and a large number of individuals are going back to their hometowns and villages. Traders in Maharashtra conducted protests against a potential lockdown, fearing that their daily activities would be totally stopped in the event that a lockdown would be 
reimposed. It's highly possible that the same situation that India faced exactly a year ago is back, with even worse implications. It is at this time that mental health needs need to be taken care of. The situation poses a grim threat that is far worse than the second wave. The absence of crowd control measures, incorrect wearing of masks, and COVID-19 numbers rising every day are some of the facets of anxiety that have sprung up once again in the second wave. Learning from the lockdown experience a year ago, it is important that the mental health of citizens be taken care of [23].

Thus there are various facets of anxiety seen during COVID-19 that need to be addressed and mental health professionals need to be cognizant of the various social, psychological and environmental factors that fuel these anxieties.

\section{REFERENCES}

1. Pfefferbaum B, North CS. Mental health and the Covid-19 pandemic. New Engl J Med 2020;383:510-2.

2. Cullen W, Gulati G, Kelly BD. Mental health in the Covid-19 pandemic. QJM: An International Journal of Medicine 2020;113:311-2.

3. Shanafelt T, Ripp J, Trockel M. Understanding and addressing sources of anxiety among health care professionals during the COVID-19 pandemic. JAMA 2020;323:2133-4.

4. Wiederhold BK. Using social media to our advantage: alleviating anxiety during a pandemic. Cyberpsychol Behav Soc Network 2020;23:197-8.

5. Asmundson GJ, Taylor S. How health anxiety influences responses to viral outbreaks like COVID-19: What all decision-makers, health authorities, and health care professionals need to know. J Anxiety Disord 2020;71:102211.

6. Trougakos JP, Chawla N, McCarthy JM. Working in a pandemic: Exploring the impact of COVID-19 health anxiety on work, family, and health outcomes. J Applied Psychol 2020;105(11):1234-8.

7. Brooks SK, Webster RK, Smith LE, Woodland L, Wessely S, Greenberg N, Rubin GJ. The psychological impact of quarantine and how to reduce it: rapid review of the evidence. Lancet 2020;395:912-20.

8. Liu CH, Zhang E, Wong GT, Hyun S. Factors associated with depression, anxiety, and PTSD symptomatology during the COVID-19 pandemic: Clinical implications for US young adult mental health. Psychiatr Res 2020;290:113172.

9. Wahba AA. Impact of COVID 19 on patient with obsessive compulsive disorder. Int Egyptian J Nurs Sci Res 2021;1:36-9.

10. Nicomedes CJ, Avila RM. An analysis on the panic during COVID-19 pandemic through an online form. J Affect Disord 2020;276:14-22.

11. De Sousa A, Mohandas E, Javed A. Psychological interventions during COVID-19: challenges for low and middle income countries. Asian J Psychiatry 2020;51:102128.

12. De Sousa A, D'souza R. Embitterment: The Nature of the Construct and Critical Issues in the Light of COVID-19. Healthcare 2020;8:304-11.

13. Menzies RE, Neimeyer RA, Menzies RG. Death Anxiety, Loss, and Grief in the Time of COVID-19. Behav Change 2020;37:111-5.

14. Girdhar R, Srivastava V, Sethi S. Managing mental health issues among elderly during COVID-19 pandemic. J Geriatr Care Res 2020;7:32-5.

15. Malathesh BC, Das S, Chatterjee SS. COVID-19 and domestic violence against women. Asian J Psychiatry 2020;53:102227.

16. Connor J, Madhavan S, Mokashi M, Amanuel H, Johnson NR, Pace LE, Bartz D. Health risks and outcomes that disproportionately affect women during the Covid-19 pandemic: A review. Soc Sci Med 2020;9:113364.

17. Khoury JE, Atkinson L, Bennett T, Jack SM, Gonzalez A. COVID-19 and mental health during pregnancy: The importance of cognitive appraisal and social support. J Affect Disord 2021;282:1161-9.

18. Moyer CA, Compton SD, Kaselitz E, Muzik M. Pregnancy-related anxiety during COVID-19: a nationwide survey of 2740 pregnant women. Arch Wom Ment Health 2020;9:1-9.

19. Liu JJ, Bao Y, Huang X, Shi J, Lu L. Mental health considerations for children quarantined because of COVID-19. Lancet Child Adolesc Health 2020;4:347-9.

20. Gupta T, Nebhinani N. Impact of COVID-19 pandemic on child and adolescent mental health. J Indian Assoc Child Adolesc Ment Health 2020;16:1-6.

21. Singh P, Singh S, Sohal M, Dwivedi YK, Kahlon KS, Sawhney RS. Psychological fear and anxiety caused by COVID-19: Insights from Twitter analytics. Asian J Psychiatry 2020;54:102280.

22. Mann FD, Krueger RF, Vohs KD. Personal economic anxiety in response to COVID-19. Personal Individ Diff 2020;167:110233.

23. Peretti-Watel P, Alleaume C, Léger D, Beck F, Verger P, COCONEL Group. Anxiety, depression and sleep problems: a second wave of COVID-19. Gen Psychiatry 2020;33(5).

Acknowledgements - Nil; Source of Funding - Nil; Conflict of Interest - Nil 